\title{
Violência sexual na região do ABC Paulista: retrato de 142 casos
}

\author{
Sexual violence in the ABC Paulista: portrait of 142 cases
}

Josyandra Paula de Freitas Rosa ${ }^{1}$, Manuella Missawa de Oliveira ${ }^{1}$, Manoel Messias de Oliveira Filho ${ }^{1}$, César Eduardo Fernandes ${ }^{1}$, Emerson Oliveira ${ }^{1}$

'Departamento de Obstetrícia e Ginecologia, Faculdade de Medicina do ABC (FMABC) - Santo André (SP), Brasil.

DOI: https://dx.doi.org/10.7322/abcshs.v43i1.994

\section{RESUMO}

Introdução: O abuso sexual deixa sequelas graves, como limitações sociais. Objetivo: Descrever o perfil das vítimas de violência sexual atendidas em um serviço especializado, na cidade de Mauá, São Paulo, entre 2008 a 2009. Métodos: Analisou-se 138 mulheres, assistidas em um Hospital Universitário. Estudo retrospectivo com levantamento de prontuários para coleta de dados. As variáveis estudadas foram: idade, etnia, relacionamento conjugal e sexual, hora da violência, número de agressores, busca por auxílio médico, uso de medicações e exames protocolares, comunicação por parte da vítima às autoridades competentes, uso de arma pelo agressor e número de gestações decorrentes. Resultados: A média de idade foi de 22 anos. A maioria não possuía relação estável, tinha atividade sexual previamente à violência, etnia branca, procurou auxílio médico em até 72 horas após o ocorrido usou medicações protocolares. No período noturno ocorreram mais crimes e a violência nesse momento teve maior probabilidade de ser praticada por mais de um agressor. Apenas 26,7\% pacientes reconheceram os agressores e somente $42,9 \%$ e $21,8 \%$ das mulheres fizeram boletim de ocorrência e exame de corpo de delito, respectivamente. Em 40,8\% foi utilizado algum tipo de arma na abordagem ou durante o crime. Por fim, nenhuma das pacientes que fez contracepção de emergência engravidou. Somente quatro engravidaram em decorrência da agressão. Conclusão: A caracterização das pessoas que sofrem violência sexual é de extrema importância para a criação de estratégias de atendimento para a profilaxia de doenças sexualmente transmissíveis e seguimento ambulatorial até finalizar o tratamento, além de acompanhamento psicológico.

Palavras-chave: violência; delitos sexuais; estupro; direitos da mulher; serviços de saúde da mulher.

\begin{abstract}
Introduction: Sexual abuse leaves severe sequels, such as social limitations. Objective: To investigate aspects of sexual violence and the victims treated at a specialized service in the city of Mauá, São Paulo, between 2008 to 2009. Methods: We analyzed 138 women, assisted at a University Hospital. Study is retrospective with survey of medical records for data collection. We studied: age, ethnicity, marital and sexual relationship, time of violence, number of aggressors, search for medical assistance, use of medications and protocol examinations, communication to the competent authorities, use of the weapon by the aggressor and number of resulting pregnancies. Results: The mean age was 22 years. Most had no stable relationship, had sexual activity before the violence, were of white ethnicity, sought medical help within 72 hours after the event and used protocol medications. At night there were more crimes and violence at that time was more likely to be committed by more than one perpetrator. Only $26.7 \%$ of the patients acknowledged the perpetrators and only $42.9 \%$ and $21.8 \%$ of the women did report bullying and examination of the body of crime, respectively. In $40.8 \%$ some kind of weapon was used in the approach or during the crime. Finally, none of the patients who did emergency contraception became pregnant. Conclusion: The characterization of people who suffer sexual violence is of extreme importance for the creation of strategies for the care of prophylaxis of sexually transmitted diseases and outpatient follow-up until the end of treatment, as well as psychological counseling.
\end{abstract}

Keywords: violence; sex offenses; rape; women's rights; women's health services. 


\section{INTRODUÇÃO}

A violência sexual contra o ser humano, fundamentalmente contra a mulher e a criança, é um grave problema de saúde pública e atinge todas as classes sociais, etnias, culturas e religiões de todos os países no mundo. A Organização Mundial da Saúde (OMS) define violência como "o uso intencional da força física ou do poder, real ou em ameaça, contra si próprio, contra outra pessoa, ou contra um grupo ou uma comunidade, que resulte ou tenha possibilidade de resultar em lesão, morte, dano psicológico, deficiência de desenvolvimento ou privação"1. Ademais, pelo Código Penal Brasileiro, define-se estupro como "constranger alguém, mediante violência ou grave ameaça, a ter conjunção carnal ou a praticar ou permitir que com ele se pratique outro ato libidinoso"2 $^{2}$. Estima-se que aproximadamente $18 \%$ das mulheres da população geral sofram pelo menos um episódio de violência sexual durante a vida ${ }^{3,4}$.

A incidência da violência sexual está relacionada à desigualdade social, a políticas sociais equivocadas que atendem homens e mulheres desproporcionalmente, às diferenças entre sexos e à cultura de domínio e honra do sexo masculino ${ }^{5}$.

O abuso sexual é uma prática que deixa sequelas graves nas vítimas, entre as alterações, chamam à atenção as desordens psicológicas e de comportamento (ansiedade, depressão, distúrbios alimentares e abuso de drogas), morbidades ginecológicas, disfunções sexuais, gravidez indesejada, além, é claro, das doenças sexualmente transmissíveis (DSTs) ${ }^{4,6-9}$. Inegavelmente, as DSTs deterioram a qualidade de vida da mulher com sérias repercussões na saúde, tais como: infertilidade, doenças inflamatórias pélvicas e aumento do risco de contaminação pelo vírus do HIV ${ }^{10,11}$

As vítimas de violência sexual ou de outro tipo de violência têm limitações em atividades sociais e cívicas em relação às demais pessoas $^{12,13}$. As morbidades relacionadas à violência sexual acabam assumindo maiores proporções porque, em geral, os agressores não se preocupam em utilizar barreiras protetoras ${ }^{8}$. Por esse motivo, é de extrema importância que essa mulher seja assistida, no âmbito social e na preservação de sua saúde. Ainda assim, devido às sequelas agudas e crônicas, uma paciente vítima de violência sexual requer mais gastos do que uma mulher que tenha sofrido outro tipo de violência ${ }^{9}$.

Apenas em 1990 foi criado o primeiro serviço no Brasil em atendimento às vítimas de violência sexual. O Hospital Jabaquara (Dr. Artur Ribeiro de Saboya), em São Paulo, foi pioneiro em criar um protocolo de assistência as vítimas com treinamento específico para a capacitação de profissionais da saú$\mathrm{de}^{14,15}$. A partir desse momento, outros serviços foram criados e políticas se voltaram de maneira mais sólida ao assunto. Como exemplo, o Ministério da Saúde, em 2006, iniciou a implantação do Sistema de Vigilância de Violências e Acidentes (VIVA), com o objetivo de obter dados e divulgar informações sobre violências e acidentes de qualquer âmbito em qualquer nível de atendimento, incluindo a violência sexual ${ }^{16,17}$.
Em seus diversos contextos, a violência no Brasil exige políticas públicas que demandam $1,9 \%$ do Produto interno bruto (PIB) nacional $^{5}$. Além disso, 98,6\% dos municípios brasileiros declararam possuir serviços sócio-assistenciais a mulheres vítimas de abuso sexual. Menos da metade, entretanto, declararam oferecer serviços como o "Serviço de Apoio e Orientação aos Indivíduos e famílias Vítimas de Violência" (45,6\%) e o "Serviço de Enfrentamento à Violência, Abuso e Exploração Sexual de Crianças e Adolescentes e suas Famílias" $(39,0 \%)^{10}$.

A Secretaria de Política para Mulheres (SPM), em 2009, registrou de seus quase 41 mil relatos de violência contra a mulher, apenas 576 casos $(1,6 \%)$ de violência sexual ${ }^{18}$. Acredita-se que, provavelmente, ocorra grande sub-notificação dos índices de violência sexual aos órgãos públicos e diversos fatores influenciam as mulheres a não notificar à polícia sobre o ocorrido: vergonha culpa medo de exposição pública e até o fato de conhecerem o agressor, são alguns pontos que impedem a denúncia do crime ${ }^{19}$.

Diante do exposto, interessou-nos nesse estudo descrever o perfil das vítimas de violência sexual atendidas em um serviço especializado, na cidade de Mauá, São Paulo, entre 2008 a 2009.

\section{MÉTODOS}

O presente estudo foi aprovado pelo Comitê de Ética em Pesquisa da FMABC, processo número 162/2010. Foram analisadas 138 mulheres vítimas de violência sexual no município de Mauá pertencente à região do ABCD Paulista entre 2008 e 2009. Todos os casos foram assistidos em um serviço especializado.

Trata-se de um estudo do tipo epidemiológico, descritivo, retrospectivo utilizando-se do levantamento de prontuários para a coleta de dados, feito pelo pesquisador principal. As variáveis estudadas foram: idade, etnia, tipo de relacionamento conjugal e sexual, hora da violência, número de agressores, busca por auxílio médico, uso de medicações e exames protocolares, comunicação por parte da vítima às autoridades competentes (inclusive a realização de exame de corpo de delito), uso de algum tipo de arma por parte do agressor e número de gravidezes decorrentes da violência. Finalmente, as estatísticas descritivas foram realizadas para análise do estudo utilizando-se o programa Microsoft Office Excel 2007.

\section{RESULTADOS}

A média de idade das mulheres foi de 22,08 anos. A maioria das pacientes não possuía relação estável (101 mulheres - 78,7\%), tinham atividade sexual previamente à violência 107 mulheres $(75,4 \%)$ e eram de etnia branca 77 vítimas (74,2\%) (Tabela 1$)$.

O período em que mais ocorreram os crimes foi o noturno (76\%) e a violência nesse momento teve maior probabilidade de ser praticada por mais de um agressor. A maioria, 108 mulheres (76\%), procurou auxílio médico em até 72 horas após o ocorrido e 89 mulheres $(62,7 \%)$ usaram medicações protocolares. Ademais, 133 (93,7\%) fizeram os exames de rotina (protocolares) previstos (Tabela 2). 
Constatou-se um único agressor em 115 casos (80,9\%). Apenas $38(26,7 \%)$ pacientes reconheceram os agressores e somente 61 $(42,9 \%)$ e $31(21,8 \%)$ mulheres fizeram boletim de ocorrência e exame de corpo de delito, respectivamente. Em 58 casos (40,8\%) foi utilizado algum tipo de arma na abordagem ou durante o crime. Houve a prisão de apenas três $(2,1 \%)$ agressores (Tabela 2$)$.

Por fim, nenhuma das pacientes que fez contracepção de emergência engravidou. Em 69 casos houve ejaculação (48,6\%), entretanto, a minoria (seis agressores) utilizou preservativo durante o estupro. Das 138 mulheres, apenas quatro acabaram engravidando em decorrência da agressão (Tabela 3).

\section{DISCUSSÃo}

Os dados desse estudo revelam que o foco principal dos criminosos são mulheres adultas jovens e em idade reprodutiva, corroborando com os achados da literatura ${ }^{20-22}$.A literatura, ainda, mostra que a maioria das vítimas de violência sexual são pardas ou negras ${ }^{23,24}$, contrariando nossos achados que mostraram que três quartos das mulheres são brancas. Ressalva-se, entretanto, que os estudos publicados sobre a violência sexual são, em sua maioria, em países onde a maior parcela da população é de etnia negra.

Consoante os achados da literatura mundial, foi constatado que a maioria das violências ocorreram no período noturno, adotado como das $18 \mathrm{~h}$ até $6 \mathrm{~h}^{20,25}$. O período noturno fornece condições favoráveis para o crime, pois a escuridão acoberta locais ermos, há menor número de pessoas nas ruas que inibiriam tal ato e um menor patrulhamento policial. Embora tenha sido maior a participação de mais de um agressor, quando a violência foi praticada à noite, o estudo revelou que a maioria dos casos de violência sexual possuía apenas um agressor.

Tabela 1: Características das pessoas que sofreram violência sexual

\begin{tabular}{|l|c|c|}
\hline Variáveis & \multicolumn{1}{|c|}{$\mathbf{n}$} & $\%$ \\
\hline $\begin{array}{l}\text { Sexo } \\
\text { Masculino }\end{array}$ & 4 & 2,82 \\
\hline Feminino & 138 & 97,18 \\
\hline Faixa Etária (anos) & & \\
\hline$<12$ & 0 & 0,00 \\
\hline 12 a 18 & 62 & 43,66 \\
\hline 19 a 59 & 80 & 56,34 \\
\hline S60 & 0 & 0,00 \\
\hline Situação Conjugal & & \\
\hline Solteiros & 91 & 64,08 \\
\hline Casados & 16 & 11,27 \\
\hline Viúvos & 1 & 0,70 \\
\hline Amasiados & 7 & 4,93 \\
\hline Separados & 9 & 6,34 \\
\hline Não consta & 18 & 12,68 \\
\hline Cor / raça & & \\
\hline Branco & 77 & 54,23 \\
\hline Preto & 30 & 21,13 \\
\hline Pardo & 23 & 16,20 \\
\hline Amarelo & 0 & 0,00 \\
\hline Vermelho & 0 & 0,00 \\
\hline Não consta & 12 & 8,45 \\
\hline & & \\
\hline
\end{tabular}

Nos dias atuais, pelo maior acesso a informações sobre as DSTs, as mulheres violentadas procuram ajuda médica após o ocorrido na maioria das vezes. Assim, é frequente comparecerem aos centros de saúde antes de completar 72 horas desde o crime. Isso é relevante, pois, permite a realização dos procedimentos protocolares, em especial, a quimioprofilaxia e assistência psicológica. Previne-se, assim, a instalação efetiva e a evolução de DSTs, inclusive a AIDS.

Tabela 2: Características da Violência Sexual

\begin{tabular}{|c|c|c|}
\hline Variáveis & $\mathbf{n}$ & $\%$ \\
\hline Agressor & & 0,00 \\
\hline Desconhecido & 95 & 66,90 \\
\hline Conhecido & 5 & 3,52 \\
\hline Colega de trabalho & 1 & 0,70 \\
\hline Cunhado & 2 & 1,41 \\
\hline Dono da casa & 2 & 1,41 \\
\hline Ex-marido & 3 & 2,11 \\
\hline Ex-namorado & 3 & 2,11 \\
\hline Filho do patrão & 1 & 0,70 \\
\hline Morador da região & 1 & 0,70 \\
\hline Motorista do carro da escola & 1 & 0,70 \\
\hline Namorado & 1 & 0,70 \\
\hline Padrasto & 1 & 0,70 \\
\hline Pai & 3 & 2,11 \\
\hline Parente & 2 & 1,41 \\
\hline Parente do patrão & 1 & 0,70 \\
\hline Patrão & 2 & 1,41 \\
\hline Sobrinho & 1 & 0,70 \\
\hline Tio & 2 & 1,41 \\
\hline Vizinho & 3 & 2,11 \\
\hline Não quer falar & 1 & 0,70 \\
\hline Não consta & 1 & 0,70 \\
\hline \multicolumn{3}{|l|}{ Horário } \\
\hline Entre $18 \mathrm{~h}$ e $6 \mathrm{~h}$ & 103 & 72,54 \\
\hline Entre $6 \mathrm{~h}$ e $18 \mathrm{~h}$ & 39 & 27,46 \\
\hline \multicolumn{3}{|l|}{ Tipo de Agressão Sexual } \\
\hline Penetração vaginal & 78 & 54,93 \\
\hline Oral e/ou anal & 3 & 2,11 \\
\hline Ambos & 47 & 33,10 \\
\hline Nenhum & 14 & 9,86 \\
\hline \multicolumn{3}{|l|}{ Tipo de arma } \\
\hline Sem arma & 84 & 59,15 \\
\hline Arma branca & 15 & 10,56 \\
\hline Arma de fogo & 43 & 30,28 \\
\hline \multicolumn{3}{|l|}{ Quantidade de agressores } \\
\hline 1 & 115 & 80,99 \\
\hline 2 & 12 & 8,45 \\
\hline 3 ou mais & 8 & 5,63 \\
\hline Não sabe & 3 & 2,11 \\
\hline Não consta & 4 & 2,82 \\
\hline \multicolumn{3}{|l|}{ Ejaculação } \\
\hline Sim & 69 & 48,59 \\
\hline Não & 27 & 19,01 \\
\hline Não sabe & 46 & 32,39 \\
\hline \multicolumn{3}{|l|}{ Boletim de ocorrência } \\
\hline Sim & 61 & 42,96 \\
\hline Não & 81 & 57,04 \\
\hline \multicolumn{3}{|l|}{ Exame de corpo de delito } \\
\hline Sim & 31 & 21,83 \\
\hline Não & 111 & 78,17 \\
\hline
\end{tabular}


É de extrema importância que os municípios se preocupem em oferecer à população serviços de apoio às vítimas de violência sexual. A falta de acompanhamento ambulatorial aumenta a chance de DSTs ${ }^{4,26}$ Adicionalmente, o uso de contraceptivo regular não alterou o curso das DSTs associadas à violência sexual ${ }^{27,28}$.

Registra-se, ainda, que a gravidez indesejada decorrente dessa violência pode ser evitada com a correta contracepção de emergência visto que nenhuma mulher que utilizou o método engravidou. Nota-se, então, a relevância do treinamento de profissionais da saúde para a execução dos procedimentos protocolares previstos nas vítimas de violência sexual. Vários estudos demonstraram a importância da correta preparação da equipe multidisciplinar no atendimento das mulheres violentadas ${ }^{13,29}$. Sabe-se que, são necessários, cuidados específicos e efetivos para essas pacientes com o objetivo de se minimizar as comorbidades físicas e psicológicas e impedir a gravidez indesejada.

Cerca de $21 \%$ das mulheres violentadas continuam a ter desordens pós-traumáticas (depressão, ideias suicidas e falta de esperança) no primeiro mês e $3,8 \%$ possuem desordens moderadas a graves após 6 meses do ocorrido ${ }^{13}$. Entretanto, o número de centros e serviços de saúde voltados a essa questão são reduzidos, e, assim, ainda há dificuldades para as mulheres vítimas de violência sexual encontrarem o adequado suporte e tratamento ${ }^{30-32}$.

Identificamos, em nosso estudo, uma baixa taxa de notificação às autoridades $(42,9 \%)$. De acordo com a National Violence Against Women Survey (NVAWS), nos Estados Unidos da América, uma em cada cinco mulheres notifica o crime à polícia, reforçando que os dados existentes são subestimados ${ }^{4}$. Estudo da Universidade de Campinas (UNICAMP) confere também que somente cerca de $20 \%$ dos casos são denunciados ${ }^{13}$. As mulheres relatam vergonha perante a sociedade e medo do agressor quando questionadas sobre o porquê de não denunciarem tal

Tabela 3: Desfecho da Violência Sexual

\begin{tabular}{|c|c|c|}
\hline Variáveis & $\mathbf{n}$ & $\%$ \\
\hline \multicolumn{3}{|c|}{ Tempo para Procura do Serviço } \\
\hline Até $72 \mathrm{~h}$ & 108 & 76,06 \\
\hline Após 72h & 34 & 23,94 \\
\hline \multicolumn{3}{|l|}{ Medicação } \\
\hline Sim & 88 & 61,97 \\
\hline Não & 54 & 38,03 \\
\hline \multicolumn{3}{|l|}{ Gravidez } \\
\hline Não & 132 & 92,96 \\
\hline Já estava & 6 & 4,23 \\
\hline Decorrência do estupro & 4 & 2,82 \\
\hline \multicolumn{3}{|c|}{ Relação sexual anterior à violência } \\
\hline Sim & 105 & 73,94 \\
\hline Não & 37 & 26,06 \\
\hline \multicolumn{3}{|l|}{ Anti-concepção } \\
\hline Sim & 32 & 22,54 \\
\hline Não & 110 & 77,46 \\
\hline
\end{tabular}

crime; mulheres mais velhas (acima dos 60 anos) e mulheres que não possuem informações sobre sexualidade são as que menos reportam violência sexual, física ou ambos ${ }^{12}$. Elas podem não ter reconhecido que foram abusadas ou ainda que contaram o fato a pessoas próximas, as quais não deram credibilidade ao relato dessas mulheres.

A minoria acabou submetendo-se ao exame de corpo de delito, o que dificulta a notificação dos casos ao poder público para que possam ser tomadas providências equivalentes ao tamanho desse grande problema. O uso de arma é comum para inibir a vítima de proteger-se e fugir do crime. O estudo da Yale School of Medicine mostra que as mulheres mais velhas são as mais resistentes a reportar que foi usado arma de fogo na abordagem quando comparadas às mais novas ${ }^{23}$.

Nosso estudo - diferentemente da literatura - demonstrou que a minoria das vítimas $(26,7 \%)$ conhecia o criminoso. A literatura mostra que em boa parte dos casos, há o conhecimento da identidade do agressor ${ }^{33}$. Os estudos chegam a apontar que em dois terços das mulheres violentadas isso é possível ${ }^{4}$.

Sendo assim, julgamos que cada vez mais as mulheres precisam de maior acesso a informações sobre sexualidade, programas adequados de assistência a saúde (física e mental) e apoio jurídico para se sentirem mais confiantes e protegidas no momento de denunciarem o crime de violência.

Em conclusão, os casos de violência sexual que foram assistidos em um serviço especializado, na cidade de Mauá, São Paulo, entre 2008 a 2009, foram a maioria mulheres, idade média de 22,08 anos. A maioria das pacientes não possuía relação estável, tinham atividade sexual previamente à violência e eram de etnia branca. O período em que mais ocorreram os crimes foi o noturno e a violência nesse momento teve maior probabilidade de ser praticada por mais de um agressor. A maioria procurou auxílio médico em até 72 horas após o ocorrido e usou medicações protocolares. A minoria reconheceu os agressores e somente 61 e 31 mulheres fizeram boletim de ocorrência e exame de corpo de delito, respectivamente. Em 58 casos foi utilizado algum tipo de arma na abordagem ou durante o crime. Houve a prisão de três agressores. Por fim, nenhuma das pacientes que fez contracepção de emergência engravidou. Em 69 casos houve ejaculação, entretanto a minoria utilizou preservativo durante o estupro. Das 38 mulheres, apenas quatro acabaram engravidando em decorrência da agressão.

A caracterização das pessoas que sofrem violência sexual é de extrema importância para a criação de estratégias de atendimento para a profilaxia de doenças sexualmente transmissíveis e seguimento ambulatorial até finalizar o tratamento, além de acompanhamento psicológico.

O estudo é limitado por ser retrospectivo, utilizando fichas de notificações hospitalares, que algumas vezes podem não terem sido adequadamente preenchidas. Sugerimos novos estudos, para estratégias de maior notificação da agressão, proteção dessas pessoas agredidas e punição aos seus agressores. 


\section{REFERÊNCIAS}

1. Krug EG, Dahlberg LL, Mercy JA, Zwi A, Lozano R. Relatório mundial sobre violência e saúde. Genebra: Organização Mundial da Saúde; 2002.

2. Brasil. Presidência da República. Lei n. 12.015, de 7 de agosto de 2009. Altera o Título VI da Parte Especial do Decreto-Lei no 2.848, de 7 de dezembro de 1940 - Código Penal, e o art. 10 da Lei no 8.072, de 25 de julho de 1990, que dispõe sobre os crimes hediondos, nos termos do inciso XLIII do art. 50 da Constituição Federal e revoga a Lei no 2.252 , de $1^{\circ}$ de julho de 1954, que trata de corrupção de menores. Disponível em: http://www.planalto. gov.br/ccivil_03/_Ato2007-2010/2009/Lei/L12015.htm Acesso em: 29 out. 2017

3. Jones JS, Alexander C, Wynn BN, Rossman L, Dunnuck C. Why women don't report sexual assault to the police: the influence of psychosocial variables and traumatic injury. J Emerg Med. 2009;36(4):417-24.

http://dx.doi.org/10.1016/j.jemermed.2007.10.077

4. Lang DL, Sales JM, Salazar LF, Hardin JW, Diclemente RJ, Wingood GM, et al. Rape victimization and high-risksexual behaviors: longitudinal study of African-American adolescent females. West J Emerg Med. 2011;12(3):333-42.

5. Brasil. Ministério da Saúde. Secretaria de Atenção à Saúde. Departamento de Ações Programáticas Estratégicas. Prevenção e tratamento dos agravos resultantes da violência sexual contra mulheres e adolescentes. Brasília: MS; 2012.

6. Eastgate G, Van Driel ML, Lennox N, Scheermeyer E. Women with intellectual. Disabilities.A study of sexuality, sexual abuse and protection skills. Aust Fam Physician. 2011;40(4):226-30.

7. Gal G, Levav I, Gross R. Psychopathology among adults abused during childhood or adolescence. J Nerv Ment Dis.2011;199(4):222-9.

http://dx.doi.org/10.1097/NMD.0b013e31820c7543

8. Swain SN, Saggurti N, Battala M, Verma RK, Jain AJ. Experience of violence and adverse reproductive health outcomes, HIV risks among mobile female sex workers in India. BMC Public Health. 2011:11:357.

http://dx.doi.org/10.1186/1471-2458-11-357

9. Peschers UM, Du Mont J, Jundt K, Pfürtner M, Dugan E, Kindermann G. Prevalence of sexual abuse among women seeking gynecologic care in Germany. Obstet Gynecol. 2003;101(1):103-8.

10. Haydon AA, Hussey JM, Halpern CT. Childhood abuse and neglect and the risk of STDs in early adult hood. Perspect Sex Reprod Health. 2011;43(1):16-22. http://dx.doi.org/10.1363/4301611

11. Abajobir AA, Kisely S, Maravilla JC, Williams G, Najman JM. Gender differences in the association between childhood sexual abuse and risky sexual behaviours: a systematic review and metaanalysis. Child Abuse Neglect. 2017;63:249-60. https://doi.org/10.1016/j.chiabu.2016.11.023

12. Yoshida K, DuMont J, Odette F, Lysy D. Factors associated with physical and sexual violence among canadian women living with physical disabilities. Health Care Women Int. 2011;32(8):762-75. https://doi.org/10.1080/07399332.2011.555826

13. Machado CL, Azevedo RCS, Facuri CO, Vieira MJN, Fernandes AMS. Posttraumatic stress disorder, depression, and hopelessness in women who are victims of sexual violence. Int $J$ Gynaecol Obstet. 2011;113(1):58-62.

https://doi.org/10.1016/j.ijgo.2010.10.016
14. Villela WV, Lago T. Conquistas e desafios no atendimento das mulheres que sofreram violência sexual. Cad Saúde Pública. 2007;23(2):471-5.

http://dx.doi.org/10.1590/S0102-311X2007000200025

15. Oliveira PS, Rodrigues VP, Morais RLGL, Machado JC. Assistência de profissionais de saúde à mulher em situação de violência sexual: revisão integrativa. RevEnfermUFPE.2016;10(5):1828-39. http://dx.doi.org/10.5205/reuol.9003-78704-1-SM.1005201632

16. Deslandes S, Mendes CHF, Lima JS, Campos DS. Indicadores das ações municipais para a notificação e o registro de casos de violência intrafamiliar e exploração sexual de crianças e adolescentes. Cad Saúde Pública. 2011;27(8):1633-45. http://dx.doi.org/10.1590/S0102-311X2011000800018

17. Silva PA, Lunardi VL, Lunardi GL, Arejano CB, Oliveira AMN, Vasques TCS. Violência intrafamiliar contra crianças e adolescentes: análise reflexiva sobre as dificuldades e a atuação da enfermagem. J Nurs UFPE. 2014;8(7):2498-2506. http://dx.doi.org/10.5205/reuol.5927-50900-1-SM.0807suppl201439

18. Brasil. Instituto Brasileiro de Geografia e Estatística (IBGE). Síntese de Indicadores Sociais. IBGE; 2010.

19. Jones JS, Alexander C, Wynn BN, Rossman L, Dunnuck C. Why women don't report sexual assault to the police: the influence of psychosocial variables and traumatic injury. J Emerg Med.2009;36(4):417-24. http://dx.doi.org/10.1016/j.jemermed.2007.10.077

20. Brasil. Ministério da Saúde. Prevenção e tratamento dos agravos resultantes da violência sexual contra mulheres e adolescentes. $2^{\mathrm{a}}$ ed. Brasília: MS; 2005

21. Gomez AM. Sexual violence as a predictor of unintended pregnancy, contraceptive use, and unmet need among female youth in Colombia. J Womens Health (Larchmt). 2011;20(9):1349-56. http://dx.doi.org/10.1089/jwh.2010.2518

22. Cook JM, Dinnen S, O'Donnell C. Older women survivors of physical and sexual violence:a systematic review of the quantitative literature. J Womens Health (Larchmt). 2011;20(7):1075-81. http://dx.doi.org/10.1089/jwh.2010.2279

23. Guimarães JATL, Villela WV. Características da violência física e sexual contra crianças e adolescentes atendidos no IML de Maceió, Alagoas, Brasil. Cad Saúde Pública. 2011;27(8):1647-53. http://dx.doi.org/10.1590/S0102-311X2011000800019

24. Facuri CO, Fernandes AMS, Oliveira KD, Andrade TS, Azevedo RCS. Violência sexual: estudo descritivo sobre as vítimas e o atendimento em um serviço universitário de referência no Estado de São Paulo, Brasil. Cad Saúde Pública. 2013;29(5):889-98. http://dx.doi.org/10.1590/S0102-311X2013000500008

25. Oshikata CT, Bedone AJ, Faundes A. Atendimento de emergência a mulheres que sofreram violência sexual: características das mulheres e resultados até seis meses pós-agressão. Cad Saúde Pública. 2005;21(1):192-9. http://dx.doi.org/10.1590/S0102-311X2005000100021

26. Go VF, Srikrishnan AK, Salter ML, Mehta S, Johnson SC, Sivaram S, et al. Factors associated with the perpetration of sexual violence among wine-shop patrons in Chennai, India. Soc Sci Med. 2010;71(7):1277-84.

http://dx.doi.org/10.1016/j.socscimed.2010.07.005

27. Dalal K, Andrews J, Dawad S. Contraception use and associations with intimate partner violence among women in Bangladesh. J Biosoc Sci. 2012;44 (1):83-94.

http://dx.doi.org/10.1017/S0021932011000307 
28. Sandor M, Dalal K. Influencing factors on time of breastfeeding initiation among a national representative sample of women in India. Health. 2013;5(12):2169-80. http://dx.doi.org/10.4236/health.2013.512296

29. Bacchus LJ, Bewley S, Vitolas CT, Aston G, Jordan P, Murray SF. Evaluation of a domestic violence intervention in the maternity and sexual health services of a UK hospital. Reprod Health Matters. 2010;18(36):147-57. http://dx.doi.org/10.1016/S0968-8080(10)36526-8

30. Moreno CL, Morril, AC, El-BasselN. Sexualrisk factors for Hiv and violence among Puerto Rican women in new York City. Health Soc Work. 2011;36(2):87-97.

http://dx.doi.org/10.1093/hsw/36.2.87
31. Fanslow JL, Robinson EM. Physical injuries resulting from intimate partner violence and disclosure to healthcare providers: results from a New Zealand population-based study. Inj Prev. 2011;17(1):37-42

http://dx.doi.org/10.1136/ip.2009.026104

32. Trigueiro TH; Merighi MAB; Medeiros ARP; Lopes CE; Mata NDS Jesus MCP. Victims of sexual violence attended in a specialized service. Cogitare Enferm. 2015; 20(2):249-56. http://dx.doi.org/10.5380/ce.v20i2.40355

33. Souto RQ, Araújo FKDC, Xavier AFC, Cavalcanti AL. Rape against Brazilian Women: Characteristics of Victims and Sex Offenders. Iran J Public Health. 2015; 44(12):1613-9. 